\title{
EIGHT COMPONENTS OF A DESIGN THEORY OF SONIFICATION
}

\author{
Michael A. Nees \\ Lafayette College \\ Oechsle Hall \\ Easton, PA, 18040 USA \\ neesm@lafayette.edu
}

\begin{abstract}
Despite over 25 years of intensive work in the field, sonification research and practice continue to be hindered by a lack of theory. In part, sonification theory has languished, because the requirements of a theory of sonification have not been clearly articulated. As a design science, sonification deals with artifacts - artificially created sounds and the tools for creating the sounds. Design fields require theoretical approaches that are different from theory-building in natural sciences. Gregor and Jones [1] described eight general components of design theories: (1) purposes and scope; (2) constructs; (3) principles of form and function; (4) artifact mutability; (5) testable propositions; (6) justificatory knowledge; (7) principles of implementation; and (8) expository instantiations. In this position paper, I examine these components as they relate to the field of sonification and use these components to clarify requirements for a theory of sonification. The current status of theory in sonification is assessed as it relates to each component, and, where possible, recommendations are offered for practices that can advance theory and theoretically-motivated research and practice in the field of sonification.
\end{abstract}

\section{INTRODUCTION}

In 1997, The Sonification Report [2] identified the lack of a theory of sonification as a major barrier to advancement of the field. In 2011, Walker and Nees's Theory of Sonification chapter [3] reiterated these concerns while pointing to incremental progress toward theory as a reason for optimism. Yet that incremental progress seems to have stalled, and the same dilemma remains with little evident momentum toward a resolution (see [4]). Although the reasons for the lack of sustained, intensive efforts toward theory-building in sonification are unclear, two potential explanations are disciplinary differences regarding the definition, role, and value of theory, and the fledgling nature of the field. Interdisciplinarity can be viewed as a strength of the auditory display community, but different disciplinary understandings of the forms and roles of theory might impede theory development [3]. Further, systematic progress in the field only began around 30 years ago [5].

Regardless of the reasons, sonification theory remains so underdeveloped that even the path to advance theory-building for sonification remains unclear. Recently, however, sonification researchers have begun to consider how lessons learned from broader areas of inquiry in design research might be translated to the study of sonification (see [6]). Design research has developed approaches for dealing with barriers similar to those facing sonification theory. This position paper draws connections between design theory and sonification theory in an attempt to identify paths toward

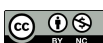

This work is licensed under Creative Commons Attribution Non Commercial 4.0 International License. The full terms of the License are available at http://creativecommons.org/licenses/by-nc/4.0 advancing sonification theory. Regarding scope, design theory is most relevant to sonification for the purposes of conveying information in human-machine interfaces, and that is the focus of this paper. Although some of the discussion presented here incidentally might be applicable to sonification as art or composition, I have not attempted to examine or elaborate those connections.

\section{STATUS OF SONIFICATION THEORY AND PRACTICE}

Vickers recently said, "I think our knowledge of sonification design and theory is still fairly primitive" (as quoted in Quinton and colleagues [4]), and this sentiment seems to be widely held among sonification experts. The sonification literature, however, has featured various attempts at theorizing - what Weick [7] described as "activities like abstracting, generalizing, relating, selecting, explaining, synthesizing, and idealizing" (pp. 389) that result in pseudotheory before fully- developed theory emerges. The sonification literature has produced scholarship with long lists of references cataloging variables and constructs [3], taxonomies [8], [9], design space maps [10], conceptual models [11], design guidelines [12], and frameworks for capturing design patterns [13], yet none of these are theories of sonification (see [14], [15]).

In some applied fields, a wealth of knowledge resides in practices that have not yet been codified formally as theory. Much has been written about gaps between theory and practice in design fields (e.g., [16]). Theoretical researchcharacteristic of academic approaches and whose purpose is to discover generalizable knowledge - has been criticized for being too abstract or removed to guide specific applications of knowledge in practice. Practice in design fields, on the other hand, is devoted to solving particular instances of immediate real-world problems and, as such, may result in one-off solutions that are not broadly shared and/or offer little contribution to re-usable knowledge. This creates a dilemma such that research discoveries may not be translated into practice (i.e., the knowledge is unknown, unused, or unusable for the practitioner), while designs used in practice may be produced on an ad hoc basis each time a problem is encountered with little awareness by the designer of why the resulting artifact was effective (or ineffective) and little concern for preserving the solution for future use by others.

In general, however, the field of sonification has been dominated by academic research. In fields characterized by theory-practice gaps, practitioner-designers solve problems in systems that are deployed or imminently will be deployed. For example, auditory alarms have been widelyused in applications for some time, and auditory alarms arguably have enjoyed the benefits of symbiotic exchanges in knowledge between research on auditory alarm design and 
information gleaned from analyzing the outcomes associated with auditory alarms as they have been used in widespread deployment (see, e.g., [17]). This example illustrates the theory-practice gap in the more traditional sense. There are few if any other examples, however, of ubiquitous deployment of sonification in practice (for a recent discussion, see [18]). Thus, for sonification, the theorypractice gap is different from the gap in other domains for which robust academic research and widespread practical applications co-exist.

The theory-practice gap in sonification as it stands currently seems to be more of a chasm between (1) academic research on potential sonification solutions to information display; (2) a (nearly complete) dearth of actual use of sonification in practice. Sonification as a field appears to be characterized to a nontrivial extent by the on-going development of sonification techniques in the absence of both generalizable theory and widespread (or any) use of sonification in practice. This type of approach-which I describe as audio for the sake of audio-produces novel sonification techniques, often without evaluation, as proofsof-concept that audio artifacts can be produced using particular processes. This work generally appears to be accomplished by academics, yet it is largely atheoretical (in that in produces one-off concept-designs rather than programmatic, generalizable knowledge) and also does not appear to be driven by need or demand for an immediate audio solution to any practical problem, even if the design space does include legitimate practical problems that could be addressed using audio. Although proof-of-concept research can offer scholarly contributions to a field, it is representative of pre-theoretical stages of inquiry [19].

\section{SONIFICATION THEORY AS DESIGN THEORY}

In this pre-theory stage, a specification of the requirements of a theory of sonification could help to provide a framework in which progress toward a theory of sonification could proceed. The formulation of a theory of sonification currently appears to be an exercise in examining potentialities rather than extant real-world conditions, which complicates our ability to begin to articulate what a theory of sonification should accomplish. To some extent, design research already has grappled with this dilemma. In trying to make the case for design as science, Simon [20] said, "The natural sciences are concerned with how things are...Design, on the other hand, is concerned with how things ought to be..." (pp. 69). Design research would seem to offer a useful launch point for specifying the requirements of a theory of sonification [6].

Design fields deal with artifacts-artificial human creations in the form of technology, so theory-building occurs in a way that is different from the way theory develops in natural sciences (e.g., [15], [20]). Design theory must explain phenomena related to the form of the artifacts themselves, the creation of artifacts, and the use of artifacts in practice. Design theory helps to ensure that research contributes to programmatic accumulation of knowledge such that: (1) research findings can be integrated into a general framework of understanding; (2) successes and best practices are carried forward and expanded upon; and (3) mistakes are not repeated.

A focus on theory-building would have several benefits across the spectrum of research and practice in the field of sonification. Venable, for example, [21] placed theory-building as the center hub of a trio of other design science activities, including (1) inventing/creating the technology; (2) defining the problem space of the technology; and (3) evaluating the technology. The sonification literature to date, has emphasized the creation of sonification as a scholarly activity, with some (but perhaps less) attention paid to defining the problem spaces for sonification and evaluating sonification's ability to meet goals within a problem spacesteps that will be imperative for sonification to be effective in practice (for a discussion, see [22]). To explain, the audio for the sake of audio approach has undertaken the creation of audio solutions under the assumption that an audio solution is necessary for some problem space - as assumption that may or may not hold across many potential applications (see, e.g., [23]). Further, only a fraction of the novel sonification approaches that have been presented have been subjected to rigorous evaluation. Sonification theory, as a central hub of activities related to inventing sonification, defining the problem space of sonification, and evaluating sonification, could help to provide the crucial link between existing activities in the field - particularly the pursuit of sonification methods and approaches - and other important but relatively under-developed activities related to evaluating the usefulness of sonification in real problem spaces for which audio may offer viable solutions.

\section{GREGOR AND JONES' ANATOMY OF A DESIGN THEORY APPLIED TO SONIFICATION}

In a highly-cited work on the requirements of a design theory, Gregor and Jones [1] synthesized multiple perspectives to derive eight essential components of design theories: (1) purposes and scope; (2) constructs; (3) principles of form and function; (4) artifact mutability; (5) testable propositions; (6) justificatory knowledge; (7) principles of implementation; and (8) expository instantiations. These components offer a relatively complete account of the meta-requirements for theory in design fields that emphasizes the unique challenges of formulating design theory. This section examines sonification theory and assesses the current completeness of sonification theory with respect to the components.

\subsection{Purpose and Scope}

Gregor and Jones [1] defined the purpose and scope of design theory as "the set of meta-requirements or goals that specifies the type of system to which the theory applies and in conjunction also defines the scope, or boundaries, of the theory" (pp. 325). The requirements enumerated in a statement of purpose and scope are "meta" in that they should generalize to all (or at least a class of) sonification artifacts rather than a particular instance.

As a useful starting point for considering the purpose and scope of a theory of sonification, The Sonification Report [2] stated, "Sonification is defined as the use of nonspeech audio to convey information. More specifically, sonification is the transformation of data relations into perceived relations in an acoustic signal for the purposes of facilitating communication or interpretation" (pp. 4, italics retained from original). Hermann [9] parsed this definition in a manner that is helpful for establishing the boundaries of a sonification theory. The set of artifacts to which a sonification theory applies are specified as nonspeech audio and implicitly the tools used to create the audio. This immediately excludes speech sounds from the scope of the theory. Nonspeech audio could include naturally occurring environmental sounds, music, etc., though the second statement further clarifies that sonification begins with data 
relations that are transformed (presumably deliberately) into perceived relations in an acoustic signal for the purposes of facilitating communication or interpretation. This further refines the scope to include only those sounds that have been deliberately created to represent relations in data for the purpose of understanding the data. Hermann further elaborated a set of four conditions that represent metarequirements for an audio artifact to fall under the purview of a theory of sonification: (1) the sound must represent "objective properties or relations in the input data"; (2) the transformation from data to sound must be systematic such that "there is a precise definition provided of how the data...caused the sound to change"; (3) the sound must be reproducible; and (4) the sonification system must be reusable with the same or different data (for a detailed discussion of these conditions, see [9]).

As such, the purpose and scope of a theory of sonification were apparent in the early definitions of sonification (e.g., [2]). Further, the field has examined and debated the boundaries of sonification (e.g., [9]). In this regard, sonification theory has achieved a degree of maturity that offers a solid grounding regarding its purpose and scope. A theory of sonification explains how, when, and why to use nonspeech sounds to convey information in systems using audio artifacts that are objective, systematic, reproducible, and reusable. To interpret further, this scope includes both audio-only and multimodal use of nonspeech sounds and excludes speech sounds, music, and incidentally occurring environmental sounds except to the extent that a consideration of these excluded factors might impact the use of nonspeech sounds to convey information. Edge cases (e.g. spearcons, see [24]) may challenge our understanding of the boundaries of a theory, and there is some ambiguity in the field about what it means to "convey information" (see section 6.5 below). Also, it is not clear if sonification could be captured in a single grand design theory, or if many related theories will be required for different uses of sonification. Thus, further refinement of the purpose and scope of sonification theory may occur in the future. Yet the purpose and scope of a theory of sonification appear to be articulated in a manner that is clear enough for mature theory to develop.

\subsection{Constructs}

Gregor and Jones [1] defined constructs as "representations of the entities of interest in the theory...these entities could be physical phenomena or abstract theoretical terms" (pp 325). Constructs in a design theory must entail a broad conceptualization of representations to capture the entities of interest. Constructs in a theory of sonification must include terms used to describe the audio artifact, terms used to describe the perception of the artifact by a listener, and terms used to describe the tasks to be undertaken by a listener. For example, a theory might explain how to use earcons (the audio artifact construct) to capture attention (a psychological construct) during monitoring (a task construct). Each construct would in turn need to be operationalized with a formal way of quantifying or identifying the construct. One could arguably extend the entities of interest in a theory of sonification to include terms used to describe the data from which the sonification is derived, etc., but those are discussed here under 6.6 below.

Even before the first ICAD conference, researchers had begun to operationalize sonification constructs such as auditory icons [25] and earcons [26]. More recently, Nees and Walker (e.g., [3], [11], [27]) have presented overviews of auditory display that taxonomize types of auditory displays, tasks to-be-accomplished with auditory displays, and listener variables to consider when designing auditory displays. de Campo's Sonification Design Space Map [10] offered a framework to define and relate the types of audio artifacts produced by sonification to one another. Early work by Barrass [28] and recent work by Verona and Peres [22] emphasized the critical role of task demands in the design of auditory displays and offered examples of how to use task analysis to precisely hone in on task constructs. Perceptual research in psychology has produced decades of literature on constructs relevant to auditory perception (see, e.g., [29], [30]). Although refinement of constructs to resolve confusions represents an on-going process in the development of a theory of sonification (see, e.g., [9], [31]) the constructs of sonification appear to be articulated in a manner that is clear enough for mature theory to develop.

\subsection{Principles of Form and Function}

Gregor and Jones [1] defined this component as "the principles that define the structure, organization, and functioning of the design product or design method...this component gives an abstract 'blueprint' or architecture for the construction of an...artifact" (pp. 326-327). In the sonification literature, Barrass described several general principles of design [32]. Specific guidelines have been provided for designing auditory alarms [33], and an international standard exists for medical device alarms [34]. A sustained critical examination of these guidelines has occurred (see [35], [36]). Guidelines exists for auditory graphs and tables ([12], [37]), earcons [38], model-based sonification [39], and general use of nonverbal sounds in interfaces [40].

Still, the available principles tend to be articulated in broad terms, and most represent an initial or preliminary attempt to codify the blueprints for sonification. For example, the standards for medical device alarms - one of the more formal and specific statements of principles of auditory design available - have been legitimately criticized for producing poor designs (e.g., [41], [42]). A lack of usable guidance is a contributor to the theory-practice gap in human-computer interaction in general [16] and in sonification specifically [43]. As such, principles and guidelines for designing sonification, though present, remain incomplete. Improved and expanded principles will be required as sonification theory develops.

\subsection{Artifact Mutability}

Simon [20] said “...a science of artificial phenomena is always in imminent danger of dissolving and vanishing" (pp. 68 ). Since sonification and its related artifacts depend upon technology, the artifacts explained in a theory of sonification have the potential to exist in a tentative state that, in some cases at least, is subject to extinction from unanticipated changes that can arrive capriciously. For example, since sonification tools generally have been created independently from mass-marketed software and hardware, updates to the infrastructure supporting the tools can render tools unusable until the developer of the tool-often one researcher or labdedicates time to updating the tool. To sustain sonification tools requires a commitment from a researcher or lab to devote resources more or less continuously toward addressing difficulties that arise from software and hardware changes over which the tool developer often has little or no control. 
This is the work required to simply keep the tools usable before any resources are devoted to substantive improvements or modifications to the tools.

As a result of these challenges, the field seems to be characterized by a proliferation of one-off, novel tools and techniques whose usable lifespan is fleeting. In fact, many of the sonification tools described in ICAD proceedings are never publicly released for use by other researchers or practitioners, much less supported and updated over time. Tools (and in some cases their associated artifacts) effectively become extinct when their developer no longer has the interest in supporting and/or resources to support the tool for other users, so designers new to sonification face considerable technical obstacles to using sound in applications (see [18], [43]). Sonification might enjoy more widespread use and deployment, which in turn would broaden the base of knowledge and feed back into the development of theory, if more general audiences (e.g., in user interface design, user experience, etc.) had access to sonification tools with sustained technical support. A consideration of the mutability of artifacts seems to be a particularly underdeveloped component of a theory of sonification.

\subsection{Testable Propositions}

A theory should create new, testable predictions. Gregor and Jones [1] argued that the most general predictions of a design theory are that the goals and purpose (see section 4.1 above) will be met when the design principles of the theory (see section 4.3 above) are applied correctly. The specificity of predictions can vary considerably across different applications of a theory, but a theory should be capable of providing a framework for guiding action and a set of criteria against which the success of that action can be judged. A mature field of inquiry will focus its scholarship efforts toward examining the testable propositions of theory to refine and qualify the theory, resolve contradictions, etc.

In the sonification literature, this component is closely related to discussions regarding how to evaluate sonifications. Bonebright and colleagues, in particular, have presented practical overviews of methods for evaluating sonifications (see [44]), and evaluation has been recognized as a critical activity for the effective design of auditory displays (e.g., [27]). Yet the issue of evaluation holds a somewhat contentious place in the field. Supper [45] has documented an epistemological rift in the auditory display community between advocates of systematic user evaluation and those who believe formal evaluation is unnecessary. Effectively the difference lies in empirical versus heuristic approaches to evaluation. Testing advocates value evidence from a representative sample of users, whereas their detractors believe that an "expert" or "trained" listener can use her knowledge as a heuristic substitute for objective evidence from formal evaluations. In general, the former perspective is more characteristic of theory-building; for example, Supper [45] identified "theoretical contextualization" as a quality desired by proponents of user testing. Heuristic evaluation can be important for the design evaluation process and can provide information that is different from formal user testing (e.g., [46]). Yet it is not clear how a field in a pre-theoretical stage could formulate broadly successful heuristics in the absence of broadly successful theory. Critics of user evaluations take the position that the intended information is obviously available to the listener in the audio artifacts they produce. Currently, the heuristic evidence that an otherwise unevaluated sonification conveyed information seems to be that the creator of the sonification believes as much, which ignores the possibility that the positive evaluation could result from welldocumented threats to validity [47]. For the foreseeable future, theory-driven approaches likely will require formal, rigorous evaluation, though a standardization of heuristic principles of evaluation for sonification could be useful.

As Gregor and Jones explained, testable propositions "can take the general form: "If a system or method that follows certain principles is instantiated then it will work, or it will be better in some way than other systems or methods" (pp. 327). A fair critique of sonification research is that it runs the risk at times of becoming an industry of designs that compare audio artifacts to other audio artifacts (or nothing at all) under the assumption that an audio approach is inherently valuable, regardless of the value added as defined by task- and goal-specific criteria (for a discussion, see [22]). Novel sonification approaches should be met with scrutiny until evidence is provided that such approaches have value for meeting the goals of sonification for a particular task (see [23]).

The act of formally testing propositions alone will not necessarily produce an adequate knowledge base for a theory of sonification, because the quality of the evidence produced by testing propositions is affected by the quality of the research undertaken. There is reason to be concerned about the quality standards of user testing in the current sonification literature. Related domains of study have recently experienced a reckoning of sorts regarding the reproducibility and replicability of their findings. The "replication crisis" in psychology has revealed methodological and statistical shortcomings that have called into question a surprisingly high amount of empirical evidence in the field (see [48]). Subdisciplines in psychology (e.g., cognitive psychology) that are somewhat aligned with sonification research (with respect to both content and typical methodologies) generally have fared better under replication scrutiny than other subdisciplines, such as social psychology (see [48], [49]). But data from studies in psychology - a field that explicitly trains students in statistics and research methods and generally requires empirical evidence (the sonification equivalent of user testing) to warrant publishable contributions - appear to be unreliable at unacceptable (or at least previously underestimated) levels.

There is evidence to suggest that interdisciplinary fields like sonification also should be concerned about research quality. As an illustrative snapshot, of the 29 papers (excluding the editor's introduction) currently archived from the 2018 ICAD conference ${ }^{1}$, roughly half $(n=15)$ presented a formal user evaluation. Of note, five papers purported to introduce a new or novel sonification approach or technique, with just two of those papers providing a formal evaluation of the new approach. In the papers reporting evaluations, the median sample size was $N=17$ (ranging from 1 to 24). Although adequate sample size depends on a number of factors, it appears that research reporting evaluations at ICAD tends to be underpowered. This is problematic not only in that null results are ambiguous (i.e., they could result from lack of effects or lack of power), but also because positive findings in underpowered research can be more likely to represent Type I (false positive) statistical errors [50].

Sample size is an imperfect surrogate for overall research quality, but as one indicator, the tendency for

\footnotetext{
${ }^{1}$ https://smartech.gatech.edu/handle/1853/60062
} 
sonification studies to be underpowered suggests there is reason for concern regarding the quality of research findings in the sonification community. Sonification researchers have yet to apply the scrutiny to their own body of evidence that is currently being applied to the base of evidence in other fields such as psychology. Given the relatively lax research standards in sonification research (e.g., empirical testing of designs is viewed as optional and small sample sizes are typical), however, it seems difficult to imagine that replication and reproducibility of findings in sonification research would fare better than psychology, and it is easy to imagine that sonification research would fare worse.

In summary, the testable propositions of a theory of sonification extend readily from the definition of the term sonification (see section 5.1). There appears to be disagreement about the value of testing, however, which has resulted in disparate evaluation approaches in the field. Given recent replicability issues in related fields such as psychology, there also is reason to be concerned about the existing knowledge base for sonification.

\subsection{Justificatory Knowledge}

Design theories draw upon existing disciplinary bases of knowledge to inform and explain design decisions. Sonification's interdisciplinary roots require a theory of sonification to draw upon relevant theories in auditory perception and cognition, music, computer science, acoustics, data science, etc. This justificatory knowledge should support a theory of sonification not only by providing guidance on how to design and implement sonification, but also by explaining why those design and implementation strategies will satisfy the goals of the theory (see [1]). To some extent, then, the adequacy of a theory of sonification will be contingent upon the adequacy of its supporting justificatory knowledge from theories in related disciplines-what Walls and colleagues [51] described as "...kernel theories from natural or social sciences which govern design requirements" (pp 42; italics retained from original).

Although a complete review of the types of justificatory knowledge that could support a sonification theory is beyond the scope of this paper, several overviews have provided markers (e.g., [2], [3]). Presumably, a theory of sonification will draw connections with related work in all three elements of the auditory display system (information, display, and listener, see [52]), and representative examples of each approach can be found in the literature. In one of the earliest examples of auditory display research, Pollack [53] applied principles of information theory to benchmark performance with auditory displays. McGookin and Brewster [54] used Bregman's Auditory Scene Analysis [55] theory to improve the recognizability of co-occurring earcons. Walker and Kramer [56] provided explicit linkages between the knowledge base of traditional psychoacoustics and auditory display. In general, a rich base of justificatory knowledge is available to support the design and implementation of sonification, but translational work remains needed to elicit relevant and useful connections with related areas of inquiry.

\subsection{Principles of Implementation}

Gregor and Jones [1] defined this component as "the means by which the design is brought into being-a process involving agents and actions" which could include "... an abstract, generic design method or development approach" (pp. 328). This is different from the component outlined in section 4.3, which described the principles for creating specific types of sonifications. For sonification theory, principles of implementation entail both (1) generic principles to guide the design cycle for sonifications; and (2) generic principles for the deployment of sonifications. There are several good examples of the former in the sonification literature, but there are few if any examples of the latter.

General descriptions of sonification design cycles exist. Barrass's [57] sonification design patterns approach provided a narrative framework for the sonification design process. Johannsen [58] described a "life cycle development of auditory displays." Anderson [59] described a decisionmaking process for designing sonification. Watson and Sanderson [60] detailed how the process of ecological interface design could be applied to the development of sonification for monitoring patients under anesthesia. Nees and Walker [27] described a process for designing auditory displays for in-vehicle technologies. Each of these approaches offered generic guidance for designing sonifications.

Guidance on how to implement sonification within existing sociotechnical ecosystems is less readily available, perhaps because there are few examples of deployments of sonification at scale. Some general implementation advice (e.g., regarding strengths and limitations of audio) was offered by Kramer [52]. Edworthy [35] has discussed the implementation of auditory alarms from a holistic, systemsthinking perspective (e.g., by considering the potential negative consequences of the proliferation of alarms across devices in real world implementation, also see [36]). Tomlinson and colleagues [61] reported on lessons learned during a two-year deployment of auditory graphs in classrooms for students with visual impairments (also see [62]). Previously, the SonEnvir project also reported lessons learned from an attempt to integrate sonification broadly into work in multiple disciplines [63]. Despite the ambitious nature of these projects, there is not currently enough evidence available to formulate generic advice on how to deploy sonifications in sociotechnical systems - particularly from a macro-ergonomics perspective that addresses social, organizational, and technical challenges in less than ideal implementation circumstances. Such advice does exist in other domains (e.g., [64]) and could serve as a model for how sonification theory might develop in this regard.

\subsection{Expository Instantiation}

Gregor and Jones [1] stated, "A realistic implementation contributes to the identification of potential problems in a theorized design and in demonstrating that the design is worth considering" (pp. 329). Their conceptualization of this component included mock-ups, prototypes, and simulationsexamples of the artifacts described and explained by the theory that help to illustrate the principles of the theory. In this regard, sonification research has produced numerous instantiations of sonifications, and this activity has been particularly valued by the sonification community. As Gregor and Jones point out, however, "If the instantiation or artifact is all that there is, rather than a theory of design...the level of knowledge is that of a craft-based discipline" (pp. 329). As sonification moves from a pre-theoretical stage to more developed theoretical positions, presumably the instantiations of sonification will be adapted to align with theoretical principles. As described above, sonification research has resulted in a proliferation of sonification examples and prototypes, so the on-going development of expository 
instantiations should remain a strength of sonification research into the future.

\section{CURRENT STRENGTHS AND WEAKNESSES OF SONIFICATION THEORIZING}

Considering sonification theory as design theory under the rubric developed by Gregor and Jones [1], some areas of strength emerge regarding the current state of sonification theory. In general, sonification research appears to have adequately articulated purposes and scope, and a shared understanding of constructs has emerged. Sufficient justificatory knowledge exists to advance sonification theory, and sonification research has produced a proliferation of potential expository instantiations. These four areas represent relative strengths for theory-building.

Several of the components appear to be relatively underdeveloped at this time. Although principles of form and function have been proposed in the sonification literature, these principles have not been widely tested and refined. Further, existing principles may be articulated at a level that is too general for designing sonifications for many practical applications (see [4], [43]). Similarly, the principles of implementation in the sonification literature have been expressed in general terms (e.g., by specifying circumstances when audio is an appropriate design choice). The lack of deployment at scale of most types of sonification has left large gaps in knowledge regarding how to implement sonification in practice, particularly with respect to organizational, social, and technical challenges that may arise. Thus, principles of form and function and principles of implementation currently have achieved a preliminary status that will need further refinement and development to advance a theory of sonification.

Our current understanding and practices appear to be especially weak for at least two of the components. Although current theorizing in the sonification literature does produce testable propositions, current research practices often leave testing and evaluation of theoretical claims optional. Further, sonification researchers have not begun to consider the reproducibility and replicability of their base of knowledge, so the quality of evaluations to date may be suspect. Related fields (e.g. psychology) have had empirical findings called into question, and the psychology literature has emphasized rigorous experimental methods and quantitative analysis moreso than the sonification literature. There is reason to be concerned that replication problems also affect the sonification literature. Finally, considerations of artifact mutability have been almost entirely absent from the sonification literature. As a design field that relies on technology in the production and delivery of its artifacts, sonification theory will need to seriously grapple with solving problems related to supporting and sustaining sonification and its tools in the face of rapidly-changing technological landscapes. Currently, many sonification tools never become available to other researchers and practitioners, and one-off tools are prone to quickly become inviable. A full consideration of the lifecycle of sonification artifacts and tools must consider design, deployment, mutability, and eventual degradation of the sounds and the tools that make them.

\section{RECOMMENDATIONS FOR THEORY- BUILDING}

A number of potential recommendations for theory-building in sonification can be gleaned from a consideration of sonification theory in the context of Gregor and Jones' [1] anatomy of design theories. Explicit consideration of each component at the outset of projects could help ensure that research advances theory.

Regarding purposes and scope, before design begins the criteria for success (i.e., the information to-beconveyed by a sonification) should be defined, and these criteria should be linked to task- and goal-specific outcomes. This process likely will involve the specification of relevant constructs. Justificatory knowledge also should be made as explicit as possible at this stage of research.

Where possible, the principles of form and function that were used in the design of a sonification should be made explicit, and successes or failures of principles should be noted explicitly. Where appropriate, new principles and suggested refinements of old principles should be offered.

Robustness against changing circumstancesespecially those related to software-appears to be a particular vulnerability of sonification. A deeper consideration of artifact mutability likely would involve stronger commitments to making sonification tools and examples (including design patterns) openly available. Repositories (e.g., Github, Open Science Framework) are a superior option to personal webpages, which often become defunct despite the best intentions of researchers at the time of creation and publication. Sustaining tools, examples, and design patterns over time likely will require a concerted effort involving collaborations across the sonification community. General or multi-purpose sonification toolkits possibly could generate broader interest (e.g., from $\mathrm{HCI} / \mathrm{UX}$ professionals) than one-off, specific tools. That interest, in turn, might increase the collective motivation and commitment of the sonification community to sustaining and regularly updating such toolkits.

To advance theory, sonification research must formally test the extent to which a sonification tool or audio artifact meets the stated purposes of sonification. To the extent that the purpose of sonification is to convey information to listeners, it is incumbent upon researchers to provide evidence that the intended information has, in fact, been conveyed. Where possible, evaluation criteria should be linked to objective real-world outcomes (clinical outcomes, benchmarking against current best practice, etc.). The specific criteria that must be met in the evaluation phase will vary across use scenarios and stages of research (early/exploratory versus advanced/confirmatory, etc.). If a particular application domain is, for example, dominated by visual displays, it seems of little use to compare one sonification prototype to another unless both are also referenced to the level of performance achieved using existing approaches or the required level of performance for a particular task while using the display. One sonification could be statistically superior to another, with both falling short of criteria related to real-world usefulness.

Sonification as a field also likely would benefit from an examination of the reproducibility of its research findings. This might include the development of formal statements regarding best practices in research methods and statistical analyses. For example, psychology has seen a push toward pre-registration of research studies, open sharing of 
research data, and reforms of statistical practices ${ }^{2}$. Further, some have begun to advocate for (and coordinate) replication studies of important findings by students as part of training ${ }^{3}$, which partially addresses the problem of lack of incentives for researchers to invest resources in replication studies. It would be in the interest of sonification researchers to follow these developments closely and adopt practices that improve research quality.

Beyond user testing - and taking into account the resistance to user testing in some quarters of the field - the development of formal heuristic forms of evaluation could potentially be of value for sonification. Useful heuristics may be difficult to derive until other areas described in this paper are developed more completely. At some point in the future when theoretical evidence has accumulated, however, a formal heuristic checklist for sonification design (like those in $\mathrm{HCI} / \mathrm{UX}^{4}$ ) could be useful.

Sonification remains mostly unexamined at any scale of implementation in practice, because significant barriers exist to implementing sonification in design [18]. In perhaps the only systematic attempt to understand how audio is viewed in design practice, Frauenberger, Stockman, and Bourguet [43] conducted a survey regarding the use of audio in interface design. Barriers included the lack of standards, lack of successful design patterns, and lack of guidance for using audio, and lack of appropriate tools for design. Research to follow-up and expand upon the questions posed by Frauenberger et al. [43] seems warranted. Ultimately, a great deal more information is needed to understand how to support the delivery of sonification across organizational, social, and technical contexts, because so little information is available about actual implementation of sonification beyond lab studies. To address this gap in knowledge likely will require sustained, coordinated efforts across multiple research labs. Indeed, overcoming many of the obstacles to the development of sonification theory likely will require intensive collaboration. From the perspective of theory development, efforts to thoroughly evaluate and technically support select promising sonifications through a deployment life cycle of actual use would seem to be more valuable than the one-off, proof-of-concept projects that have characterized a considerable proportion of research in the field to date.

\section{REFERENCES}

[1] S. Gregor and D. Jones, "The Anatomy of a Design Theory," J. Assoc. Inf. Syst., vol. 8, no. 5, pp. 313-335, May 2007.

[2] G. Kramer et al., "The Sonification Report: Status of the Field and Research Agenda. Report prepared for the National Science Foundation by members of the International Community for Auditory Display," 1999.

[3] B. N. Walker and M. A. Nees, "Theory of sonification," in Principles of Sonification: An Introduction to Auditory Display, T. Hermann, A. Hunt, and J. Neuhoff, Eds. Berlin, Germany: Logos Publishing House, 2011, pp. 9-39.

[4] M. Quinton, I. McGregor, and D. Benyon, "Investigating effective methods of designing sonifications," in Proceedings of the 24th International Conference on Auditory Display (ICAD 2018), Michigan Technological University, 2018.

[5] S. P. Frysinger, "A brief history of auditory data representation to the 1980s," in Proceedings of the 11

\footnotetext{
${ }^{2}$ https://improvingpsych.org/

${ }^{3} \mathrm{https}: / /$ osf.io/wfc6u/

${ }^{4}$ https://www.nngroup.com/articles/ten-usability-heuristics/
}

International Conference on Auditory Display (ICAD 2005), Limerick, Ireland, 2005, pp. 410-413.

[6] M. Jeon, B. N. Walker, and S. Barrass, "Introduction to the Special Issue on Sonic Information Design: Theory, Methods, and Practice, Part 1," Ergon. Des., vol. 26, no. 4, pp. 3-3, Oct. 2018.

[7] K. E. Weick, "What Theory Is Not, Theorizing Is," Adm. Sci. Q., vol. 40, no. 3, pp. 385-390, Sep. 1995.

[8] T. Letowski et al., "Human factors military lexicon: Auditory displays," Army Research Laboratory Technical Report, 2001.

[9] T. Hermann, "Taxonomy and Definitions for Sonification and Auditory Display," in Proceedings of the 14th International Conference on Auditory Display, Paris, France, 2008.

[10] A. de Campo, "Toward a sonification design space map," in Proceedings of the 13th International Conference on Auditory Display (ICAD 2007), Montreal, Canada, 2007, pp. 342-347.

[11] M. A. Nees and B. N. Walker, "Listener, task, and auditory graph: Toward a conceptual model of auditory graph comprehension," in Proceedings of the 13th International Conference on Auditory Display (ICAD 2007), Montreal, Canada, 2007, pp. 266-273.

[12] L. M. Brown, S. A. Brewster, S. A. Ramloll, R. Burton, and B. Riedel, "Design guidelines for audio presentation of graphs and tables," in Proceedings of the 2003 International Conference on Auditory Display, Boston, MA, 2003.

[13] C. Frauenberger, T. Stockman, and M. L. Bourguet, "Pattern Design in the Context Space A Methodological Framework for Auditory Display Design," in Proceedings of the 13th International Conference on Auditory Display (ICAD 2007), Montreal, Canada, 2007.

[14] R. Sutton and B. Staw, "What Theory is Not," Adm. Sci. Q., vol. 40, no. 3, pp. 371-384, 1995.

[15] J. Hooker, "Is Design Theory Possible?," J. Inf. Technol. Theory Appl. JITTA, vol. 6, no. 2, Jul. 2004.

[16] L. Colusso, C. L. Bennett, G. Hsieh, and S. A. Munson, "Translational Resources: Reducing the Gap Between Academic Research and HCI Practice," in Proceedings of the 2017 Conference on Designing Interactive Systems, New York, NY, USA, 2017, pp. 957-968.

[17] J. Edworthy, "Medical audible alarms: A review," J. Am. Med. Inform. Assoc., vol. 20, no. 3, pp. 584-589, May 2013.

[18] M. A. Nees, "Auditory Graphs Are Not the 'Killer App' of Sonification, But They Work," Ergon. Des., vol. 26, no. 4, pp. 25-28, Oct. 2018.

[19] S. Gregor and A. R. Hevner, "Positioning and presenting design science research for maximum impact," MIS $Q$., pp. 337-355, 2013.

[20] H. A. Simon, "The Science of Design: Creating the Artificial," Des. Issues, vol. 4, no. 1/2, pp. 67-82, 1988.

[21] J. Venable, "The role of theory and theorising in design science research," in Proceedings of the 1st International Conference on Design Science in Information Systems and Technology (DESRIST 2006), 2006, pp. 1-18.

[22] D. Verona and S. C. Peres, "A Comparison between the Efficacy of Task-Based Vs. Data-Based sEMG Sonification Designs," in The 23rd International Conference on Auditory Display (ICAD 2017), Pennsylvania State University, 2017.

[23] J. Edworthy, "Does sound help us to work better with machines? A commentary on Rautenberg's paper 'About the importance of auditory alarms during the operation of a plant simulator," Interact. Comput., vol. 10, pp. 401-409, 1998. 
[24] B. N. Walker et al., "Spearcons (Speech-Based Earcons) Improve Navigation Performance in Advanced Auditory Menus," Hum. Factors, vol. 55, no. 1, pp. 157-182, 2013.

[25] W. W. Gaver, "Auditory Icons: Using Sound in Computer Interfaces," Human-Computer Interact., vol. 2, no. 2, pp. 167-177, Jun. 1986.

[26] M. M. Blattner, D. A. Sumikawa, and R. M. Greenberg, "Earcons and Icons: Their Structure and Common Design Principles," Human-Computer Interact., vol. 4, no. 1, pp. 1144, Mar. 1989.

[27] M. A. Nees and B. N. Walker, "Auditory displays for invehicle technologies," in Reviews of Human Factors and Ergonomics, P. Delucia, Ed. Thousand Oaks, CA: Sage Publishing/Human Factors and Ergonomics Society, 2011, pp. 58-99.

[28] S. Barrass, "TaDa! demonstrations of auditory information design," presented at the Proceedings of the 1996 International Conference on Auditory Display, 1996.

[29] J. G. Neuhoff, Ecological Psychoacoustics. New York: Academic Press, 2004.

[30] C. L. Baldwin, Auditory Cognition and Human Performance: Research and Applications. Boca Raton, FL: CRC Press, 2012.

[31] M. A. Nees, "Have we forgotten auditory sensory memory? Retention intervals in studies of nonverbal auditory working memory," Front. Psychol., vol. 7, 2016.

[32] S. Barrass, "Some golden rules for designing auditory displays," in Csound Textbook, Cambridge, MA: MIT Press, 1998.

[33] R. D. Patterson, "Guidelines for auditory warning systems on Civil Aircraft," 1982.

[34] I. E. Commission and others, IEC 60601-1-8: Medical electrical equipment-General requirements, tests and guidance for alarm systems in medical electrical equipment and medical electrical systems. Geneva, Switzerland: Author, 2005.

[35] J. Edworthy, "The design and implementation of nonverbal auditory warnings," Appl. Ergon., vol. 25, no. 4, pp. 202-210, Aug. 1994.

[36] J. Edworthy and E. Hellier, "Fewer but better auditory alarms will improve patient safety," Br. Med. J., vol. 14, no. 3, pp. 212-215, 2005.

[37] J. H. Flowers, "Thirteen years of reflection on auditory graphing: Promises, pitfalls, and potential new directions," in Proceedings of the 11 International Conference on Auditory Display (ICAD 2005), Limerick, Ireland, 2005.

[38] S. A. Brewster, P. C. Wright, and A. D. Edwards, "Experimentally derived guidelines for the creation of earcons," in Adjunct Proceedings of HCI, 1995, vol. 95, pp. $155-159$.

[39] T. Hermann, "Model-based sonification," in The Sonification Handbook, 2011, pp. 399-427.

[40] J. Hereford and W. Winn, "Non-speech sound in humancomputer interaction: A review and design guidelines," $J$. Educ. Comput. Res., vol. 11, pp. 211-233, 1994.

[41] A. N. Wee and P. M. Sanderson, "Are melodic medical equipment alarms easily learned?," Anesth. Analg., vol. 106, no. 2, pp. 501-507, 2008.

[42] P. Lacherez, E. L. Seah, and P. M. Sanderson, "Overlapping melodic alarms are almost indiscriminable," Hum. Factors, vol. 49, no. 4, pp. 637-645, 2007.

[43] C. Frauenberger, T. Stockman, and M.-L. Bourguet, "A survey on common practice in designing audio user interface," in Proceedings of the 21st British HCI Group Annual Conference on People and Computers, 187-194, 2007.
[44] T. L. Bonebright and J. H. Flowers, "Evaluation of auditory displays," in The Sonification Handbook, T. Hermann, A. Hunt, and J. G. Neuhoff, Eds. Logos Verlag Berlin, Germany, 2011.

[45] A. Supper, "'Trained ears' and 'correlation coefficients': A social science perspective on sonification," in Proceedings of the 18th International Conference on Auditory Display (ICAD 2012), Atlanta, GA, 2012

[46] W. Tan, D. Liu, and R. Bishu, "Web evaluation: heuristic evaluation vs. user testing.," Int. J. Ind. Ergon., vol. 39, no. 4, pp. 621-627, 2009.

[47] D. T. Campbell and J. C. Stanley, Experimental and quasi-experimental designs for research. Ravenio Books, 2015.

[48] O. S. Collaboration, "Estimating the reproducibility of psychological science," Science, vol. 349, no. 6251, p. aac4716, Aug. 2015

[49] G. Mitchell, "Revisiting Truth or Triviality: The External Validity of Research in the Psychological Laboratory," Perspect. Psychol. Sci., vol. 7, no. 2, pp. 109-117, Mar. 2012.

[50] K. S. Button et al., "Power failure: Why small sample size undermines the reliability of neuroscience," Nat. Rev. Neurosci., vol. 14, no. 5, pp. 365-376, May 2013.

[51] J. G. Walls, G. R. Widmeyer, and O. A. El Sawy, "Building an information system design theory for vigilant EIS," Inf. Syst. Res., vol. 3, no. 1, pp. 36-59, 1992.

[52] G. Kramer, "An introduction to auditory display," in Auditory Display: Sonification, Audification and Auditory Interfaces, SFI Studies in the Sciences of Complexity, Proceedings, 1994, pp. 1-77.

[53] I. Pollack, "The information of elementary auditory displays," J. Acoust. Soc. Am., vol. 24, no. 6, pp. 745-749, 1952.

[54] D. K. McGookin and S. A. Brewster, "Understanding concurrent earcons: Applying auditory scene analysis principles to concurrent earcon recognition," ACM Trans. Appl. Percept. TAP, vol. 1, no. 2, pp. 130-155, 2004.

[55] A. S. Bregman, Auditory Scene Analysis: The Perceptual Organization of Sound. Cambridge, MA: MIT Press, 1990.

[56] B. N. Walker and G. Kramer, "Ecological psychoacoustics and auditory displays: Hearing, grouping, and meaning making," in Ecological psychoacoustics, J. Neuhoff, Ed. New York: Academic Press, 2004, pp. 150-175. [57] S. Barrass, "Sonification design patterns," Jul. 2003.

[58] G. Johannsen, "Auditory displays in human-machine interfaces,” Proc. IEEE, vol. 92, no. 4, pp. 742-758, 2004.

[59] J. Anderson, "Creating an empirical framework for sonification design,” Jul. 2005.

[60] M. Watson and P. M. Sanderson, "Designing for Attention With Sound: Challenges and Extensions to Ecological Interface Design," Hum. Factors, vol. 49, no. 2, pp. 331-346, Apr. 2007.

[61] B. J. Tomlinson, J. Batterman, Y. C. Chew, A. Henry, and B. N. Walker, "Exploring Auditory Graphing Software in the Classroom: The Effect of Auditory Graphs on the Classroom Environment," ACM Trans Access Comput, vol. 9, no. 1, p. 3:1-3:27, Nov. 2016.

[62] S. M. Hetzler and R. M. Tardiff, "The three 'R's: Real students in real time doing real work learning calculus," Montreal, Canada, 2007.

[63] A. de Campo, C. Frauenberger, K. Vogt, A. Wallisch, and C. Daye, "Sonification as an interdisciplinary working process," Jun. 2006

[64] C. Shupe and R. Behling, "Developing and implementing a strategy for technology deployment," Inf. Manage., vol. 40, no. 4 , p. 52, 2006. 